\title{
FINE STRUCTURE OF THE LIVER IN DUBIN-JOHNSON SYNDROME
}

\author{
KYUICHI TANIKAWA \\ The Second Department of Medicine, Kurume University \\ School of Medicine, Kurume, Japan.
}

(Received for publication August 26, 1965)

The increasing use of liver biopsy technique in recent years, has caused Dubin-Johnson syndrome to become a relatively common disease and much attention has been paid to the characteristic pigment granules in the liver cell and its relationship to jaundice.

This report is devoted to the electron microscopic observation of the liver in this disease with a special attention given to the changes of the excretory organelles, and a discussion concerning the mechanism of jaundice in this disease.

\section{MATERIALS AND METHODS}

Liver biopsy specimens taken from three patients were diagnosed as Dubin-Johnsou syndrome by their histological findings indicating characteristic pigment granules in the liver cells. In every cases there was a history of recurrent episodes of jaundice at an early age. Serum bilirubin level at the time of biopsy was $4.05 \mathrm{mg}$ (direct: $3.09 \mathrm{mg}$ ) in Case $1,1.2 \mathrm{mg}$ (direct: $1.0 \mathrm{mg}$ ) in Case 2, and $3.2 \mathrm{mg}$ (direct: $2.2 \mathrm{mg}$ ) in Case 3, and B.S.P retention was increased in all cases.

For electron microscopy, the liver biopsy specimens were fixed in a cold 1 per cent osmium tetroxide solution, buffered to $\mathrm{PH} 7.4$ with Sodium Veronal acetate. The specimens were then dehydrated in graded alcohols and embedded in Epon 812. Ultra-thin sections were cut with glass knife on a Porter-Blum microtome, stained with uranyl acetate, and examined under a HU-11 electron microscope.

\section{RESULTS}

1) Light microscopic observation (Fig. 1)

Liver cells contained numerous brown pigment granules, mainly in the centrolobular area. These granules were also observed in Kupffer cells. Liver cell cords were well preserved and no abnormalities were noted except the deposition of pigment granule.

2) Electron microscopic observation (Figs. 2. 3. 4.)

EIectron microscope revealed the characteristic pigment granules of this disease to be round or oval, electron dense granules, sized from $0.5 \mu$ to $2.5 \mu$ in diameter, 


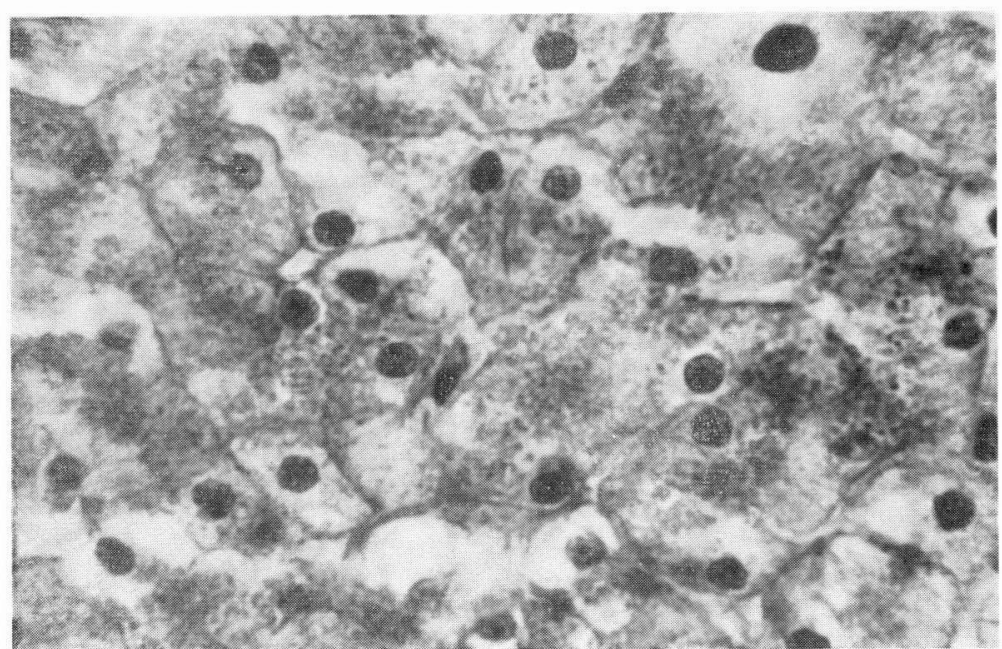

Fig. 1. Light micrograph.

The liver cells contain numerous brown pigment granules, mainly seen in the centrolobular area. These granules are also observed in the Kupffer cells.

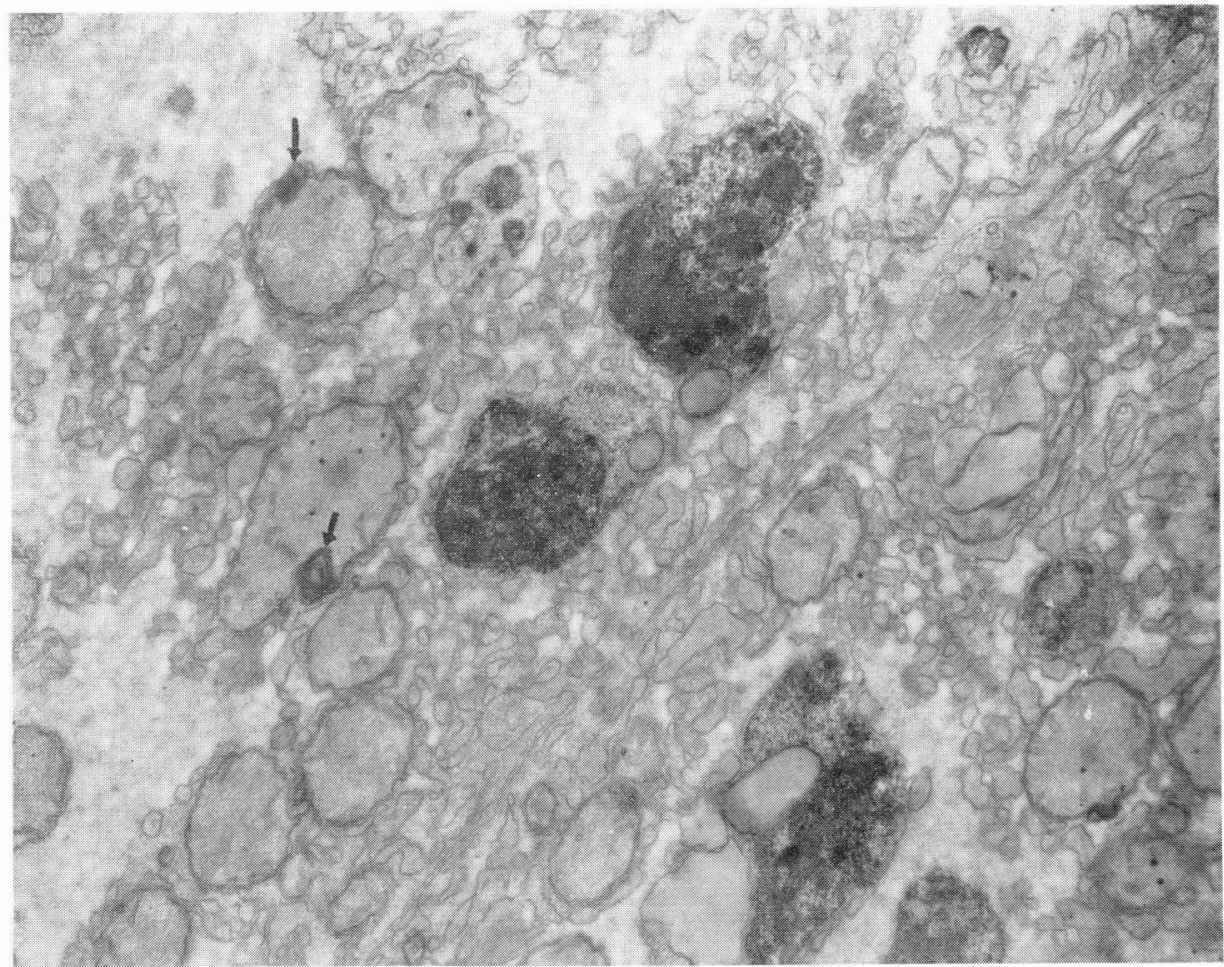

Fig. 2. Electron micrograph. $\times 24000$.

Four large electron dense granules, characteristic in Dubin-Johnson syndrome, are observed in the cytoplasm of the liver cell. The matrix of these granules contains many ferritin-like grains with larger dense particles and vacuoles of various sizes. Some mitochondria show to have myelin-like structure or dense particles (at arrows). Most of the endoplasmic reticulums are agranular and the rough surfaced are relatively decreased in number. 


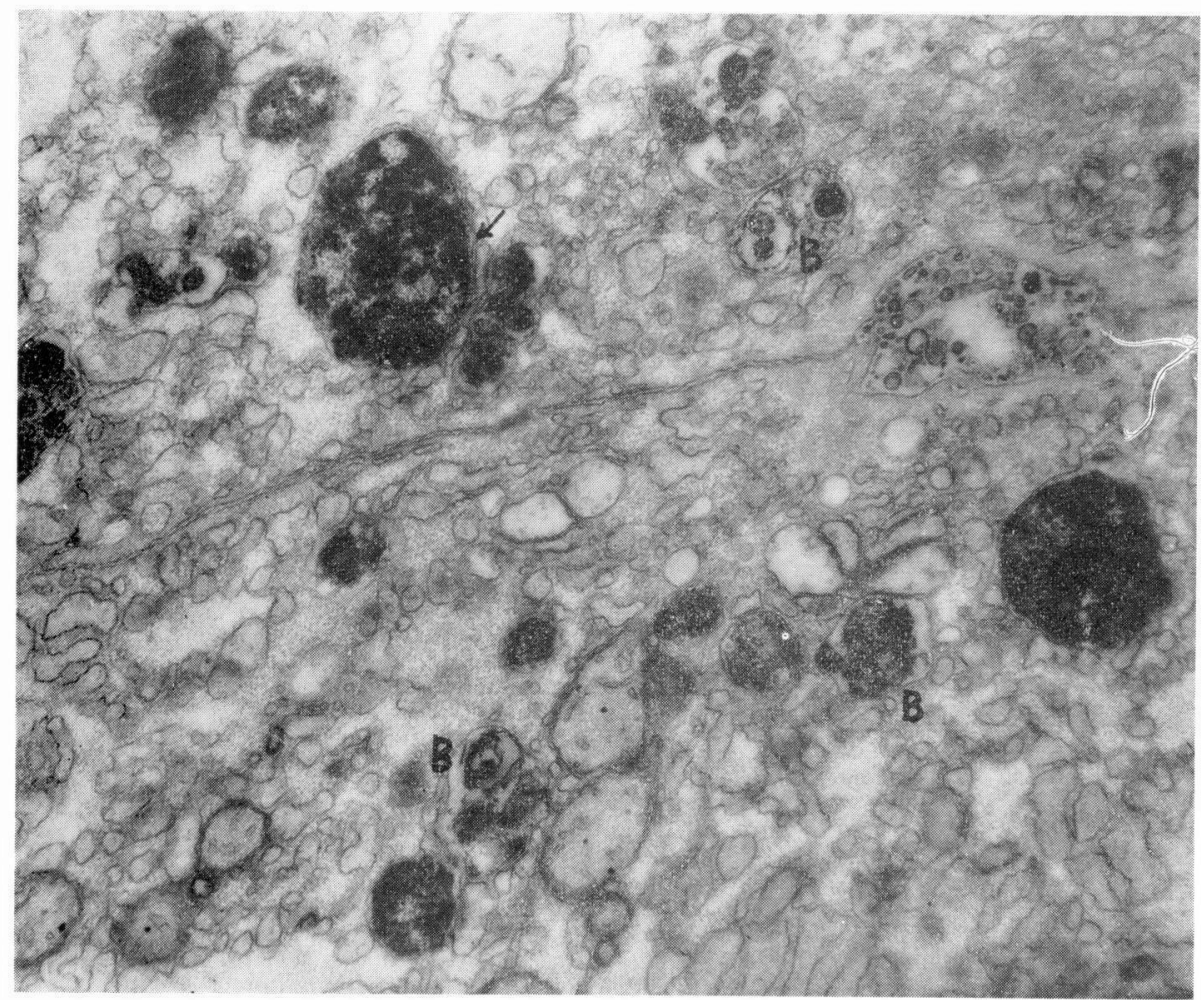

Fig. 3. Electron micrograph. $\times 18000$.

One of the dense granules of Dubin-Johnson syndrome is surrounded by double membranes (at arrow). Many bile pigment granules, in vacuoles with laminated appearance (B), are observed in the cytoplasm of the liver cell. Bile canaliculus is slightly dilated with loss of its microvilli. Fine electron dense particles are seen in Golgi apparatus.

delimited by a single surrounding membrane, and distributed diffusely in the cytoplasm of the liver cell. The matrix of these granules contained a great many ferritin-like fine grains with larger dense particles and vacuoles of various sizes. These dense granules occasionally have a double surrounding membrane or a clistae-like structure in the matrix.

Another type of electron dense granules, considered as bile pigment, was also seen in the cytoplasm, mostly around the bile canaliculus. These bile pigment granules were seen in vacuoles and mostly laminated in appearance, and frequently observed in case 1 .

Mitochondria appeared to be fairly normal in appearance, however, myelin-like structure or electron dense granules were occasionally observed in the matrix. The smooth surfaced endoplasmic reticulum was relatively predominant in number and the rough surfaced appeared to be reduced in number.

About one third of the bile canaliculi were more or less dilated with stunt or loss of their microvilli and a dense substance was frequently seen in the lumen of the canaliculus, However, rupture of terminal bar or communication between the bile 


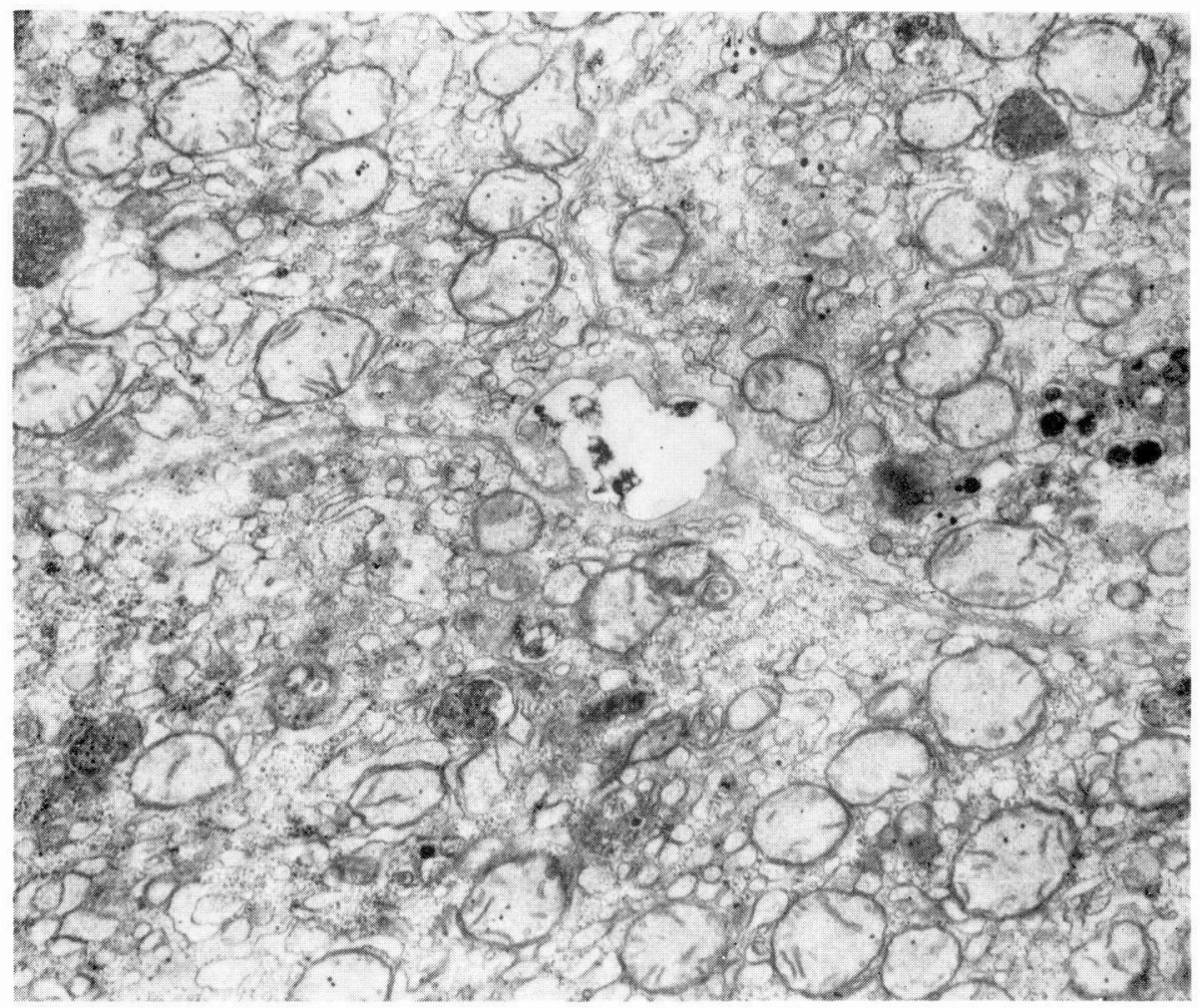

Fig. 4. Electron micrograph. $\times 13000$.

Bile canaliculus is dilated with complete loss of its microvilli and electron dense fine particles are seen in the lumen.

canalicuelus and the Disse's space was not noted. The Golgi apparatus appeared to be decreased in number and frequently contained dense fine particles in it.

\section{DISCUSSION}

The nature of the characteristic pigment granules of the Iiver cell in DubinJohnson syndrome has not been elucidated. Most of the histochemical studies have indicated that they are probadly lipofuscin or lipochrome-like substane, but some reports (1) (2) state the granules seem to resemble melanin. Histochemistry of pigments in human tissue has not been thoroughly established and it seems to be difficult in identify the nature by this technique: biochemical analysis of these granules after isolation by high power centrifugation of the liver homogenate would yield more information on their nature.

Morphological characteristics of these granules have been fully described by Miwa $^{(6)}$, Kobayashi( ${ }^{(7)}$ and Ichida ${ }^{(5)}$, and our observations were similar to their reports. These is a controversy in the morphological relationship of the organelles and these 
characteristic granules in the Dubin-Johnson syndrome. Novikoff $f^{(3.4)}$ and others ${ }^{(5)}$ reported a close relationship of these granules to the lysosome because of their acid phosphatase activity. However, others ${ }^{6.7)}$ have paid more attention to the mitochondria because of the double surrounding membrane or clistae-like structure in the granules which are found occasionally. Recently, Hunter ${ }^{8)}$ found the granules to have completely disappeared after an infection of the liver by hepatitis virus, however, they reappeared three months later. It is regrettable that an electron microscopic study was not done on this patient, for it may have been possible to determine the organelles from which these granules reappeared.

Dubin $^{9)}$ reported no obvious increase of such granules in the liver cell during the course of the disease. This finding suggests that the granules should be eliminated either via blood stream or bile in order to be in dynamic equilibrium. Our observations revealed highly electron dense substance in the bile canaliculus and Golgi apparatus, suggesting a passible route of excretion of the granules.

In our cases, especially in the one with a high grade of hyperbilirubinemia, bile pigment granules were frequently observed in the cytoplasm of the liver cell containing the characteristic granules of the Dubin-Johnson syndrome. Bile pigment granules are easily distinguished morpholofically from the granules of the Dubin-Johnson syndrome, since they are mainly located around the bile canaliculus and appear to be mostly laminated in vacuoles. Deposition of bile pigment granules in the cytoplasm implies intracellular cholestasis, and such intracellular cholestasis is commonly seen in intra- and extrahepatic obstructive jaundice ${ }^{11.12 .13)}$ and the degree of intracellular cholestasis is known to be well correlated with the grade of hyperbilirubinemia. ${ }^{10.11)}$

Frequent observation of altered bile canaliculi is important in the etiological consideration. These changes have been mentioned in some reports ${ }^{6.7)}$, but withceut too much attention. Though it is not definite whether these canalicular alterations are due to congenital fragility or secondary change, such changes are highly suggestive of impaired bile excretion.

Schoenfield ${ }^{14)}$ demonstrated a defect in hepatic excretion of bilirubin in this disease by a bilirubin load test. It has been well established that B.S.P retention is increased in this disease, but the conjugating mechanism has been unimpaired. ${ }^{14.15)}$

These findings by morphological, physiological or biochemical studies suggest that the main defect of this disease is in the excretion mechanism. It seems reasonable to postulate from the present observation, that because of relative inability of excretion due to the impaired functions of the bile canaliculus and other excretory organelles, the reflux of conjugated bilirubin, after stagnation in the liver cell, to the blood stream, is an important factor in the development of jaundice in this disease.

\section{SUMMARY}

The liver biepsy specimens taken from three patients with the Dubin-Johnson syndrome were studied under electron microscope. About one third of the bile canaliculi were found to be more or less dilated with stunt or loss of the microvilli. Bile pigment granules were frequently observed in the cytolasm of the liver cell containing the characteristic granules of the Dubin-Johnson syndrome. The mechanism of jaundice in this disease has been discussed in the light of these observations, 


\section{REFERENCES}

1) Bynum, W. T. : Mavero hepatic icterus (black liver jaundice). Gastroenterology, $33: 97$, 1957.

2) Masuda, M., вт aL. : Dubin-Johnson syndrome. Saishin.igaku, 18:1087, 1963.

3) Novikgff, A. B. and Essner, E., : The liver cell. Some new approaches to its study. Am. J. Med. 29: 101, 1960.

4) Essner, E. and Novikoff, A. B. : Human hepatocellular pigments and lysosomes. J. Ultrastruct. Res. 3: 374, 1960.

5) ICHIDA, F. ANd Funahashi, H. : Electron microscopic observation on the liver cells of cases. with Dubin-Johnson syndrome. Acta Hepato-spleno-logica, 11:332, 1964.

6) Mrwa, S, ET AL. : Dubin-Johnson syndrome. Jap. J. Gastroenterol, 55 : 691, 1958.

7) Kobayashi, J. : Electron microscopic findings in parenchymal liver cells of Dubin-Johnson syndrome with special reference to pigment granules in liver cells and morphological study for its jaundice. Jap. J. Gastroenterol. 59: 209, 1962.

8) Hunter, F. M. et AL. : Hepatitis with resulting mobilation of hepatic pigment in a. patient with Dubin-Johnson syndrome. Gastroenterology, 47 : 631, 1964.

9) Dubin, I. N., : Chronic idiopathic jaundice: A review of fifty cases. Am. J. Med., 24: 268, 1958.

10) Tanikawa, K : Elecrton microscopic observation of cholestasis in viral hepatitis with special attension to the mechanism of jaundice. proceeding of II world congress of gastroenterology, Munich 1962, III : 189.

11) Tanikawa, K. : Pathogenesis of intrahepatic obstructive jaundice. Saishin-igaku. 18: 10051963.

12) Sasaki, H. and Ichida, F., : Electron microscopic studies of cholestasis. Ann. Rep. Insit. Virus Res. 4: 172, 1961.

13) Electron microscopic observation on human liver during cholestasis. Orlandi, F., Acta. Hepato-splenol. 9: 155, 1962 .

14) Schoenfield, L. J. ET AL. : Studies of chronic idiopathic jaundice (Dubin-Johnson. syndrome). Gastroenterology 44: 101, 1963.

15) Metge, w., ET AL. : Bilirubin glucuronyl transferase activtiy in liver disease. J. Lab \& Clin. Med. 64:89, 1964 . 